\title{
Infections occurring during extracorporeal membrane oxygenation use in adult patients
}

\author{
Hsin-Yun Sun, MD, ${ }^{a}$ Wen-Je Ko, MD, ${ }^{b}$ Pi-Ru Tsai, RN, ${ }^{b}$ Chun-Chuan Sun, RN, ${ }^{\text {c }}$ Yin-Yin Chang, RN, ${ }^{c}$ \\ Ching-Wen Lee, MS, ${ }^{\mathrm{d}}$ and Yee-Chun Chen, $\mathrm{MD}, \mathrm{PhD}^{\mathrm{a}, \mathrm{c}}$
}

Objective: The application of extracorporeal membrane oxygenation in adults has been increasing, but infections occurring during extracorporeal membrane oxygenation use are rarely described.

Methods: We retrospectively analyzed the prospectively collected data on nosocomial infection surveillance of 334 patients aged 16 years or more undergoing their first extracorporeal membrane oxygenation for more than 48 hours at a university hospital from 1996 to 2007 for respiratory (20.4\%) and cardiac (79.6\%) support.

Results: During a total of 2559 extracorporeal membrane oxygenation days, 55 episodes of infections occurred in 45 patients (13.5\%), including 38 bloodstream (14.85 per 1000 extracorporeal membrane oxygenation days), 6 surgical site, 4 respiratory tract, 3 urinary tract, and 4 other infections. Stenotrophomonas maltophilia (16.7\%) and Candida species (14.6\%) were the predominant blood isolates. In stepwise logistic regression analysis, longer duration of extracorporeal membrane oxygenation use (odds ratio 1.003 ; 95\% confidence interval, $1.001-$ 1.005; $P=.004$ ), mechanical complications (odds ratio, 4.849; 95\% confidence interval, 1.569-14.991; $P=.006$ ), autoimmune disease (odds ratio, $6.997 ; 95 \%$ confidence interval, $1.541-31.766 ; P=.012$ ), and venovenous mode (odds ratio, 4.473; 95\% confidence interval, 1.001-19.977; $P=.050$ ) were independently associated with a higher risk for infections during extracorporeal membrane oxygenation use. Overall in-hospital mortality was $68.3 \%$, and its independent risk factors included older age (odds ratio, 1.037; 95\% confidence interval, 1.021-1.054; $P<.001$ ), neurologic complications (odds ratio, 51.153; 95\% confidence interval, 6.773386.329; $P<.001$ ), and vascular complications (odds ratio, 1.922; 95\% confidence interval, 1.112-3.320; $P<.001)$, but not infections during extracorporeal membrane oxygenation use.

Conclusions: Bloodstream infection was the most common infection during extracorporeal membrane oxygenation use. Duration of extracorporeal membrane oxygenation, mechanical complications, autoimmune disease, and venovenous mode seemed to be independently associated with infections. (J Thorac Cardiovasc Surg 2010;140:1125-32)

\section{Supplemental material is available online.}

Extracorporeal membrane oxygenation (ECMO) was first reported for adult respiratory distress syndrome in $1972 .^{1}$ Subsequently, ECMO was gradually accepted as a treatment modality for neonatal, pediatric, and adult patients with respiratory or cardiac failure who fail to respond to maximal medical therapy. Currently, more than 24,000 neonates,

\footnotetext{
From the Department of Internal Medicine, ${ }^{\mathrm{a}}$ Department of Surgery, ${ }^{\mathrm{b}}$ and Center for Infection Control, ${ }^{\mathrm{c}}$ National Taiwan University Hospital and National Taiwan University College of Medicine, Taipei, Taiwan; and Department of Biostatistics, ${ }^{\mathrm{d}}$ Graduate School of Public Health, University of Pittsburgh, Pittsburgh, Pa.

Disclosures: None.

Received for publication Oct 19, 2009; revisions received Jan 18, 2010; accepted for publication July 4, 2010; available ahead of print Aug 16, 2010.

Address for reprints: Yee-Chun Chen, MD, PhD, Division of Infectious Diseases, Department of Internal Medicine, National Taiwan University Hospital, No. 7, Chung-Shan South Road, Taipei, 100, Taiwan (E-mail: yeechunchen@gmail.com). $0022-5223 / \$ 36.00$

Copyright (C) 2010 by The American Association for Thoracic Surgery doi:10.1016/j.jtcvs.2010.07.017
}

7000 children, and 2000 adults have been treated with ECMO. $^{2}$ Although its use is relatively controversial in adults, ECMO has been gradually used as cardiac support in various clinical settings, such as postcardiotomy cardiogenic shock after cardiac surgery, ${ }^{3}$ bridge to heart transplantation, $^{4}$ fulminant myocarditis, ${ }^{5}$ and assistance for cardiopulmonary resuscitation (CPR). ${ }^{6}$

Infections occurring during ECMO use increase mortality in neonatal or pediatric populations, but whether they have a similar impact on adults has rarely been reported..$^{7-10}$ Thus, we assessed the occurrence, type, causative pathogens, and risk factors of infections during ECMO use as respiratory or cardiac support in adults at an extracorporeal life-support referral center. Risk factors associated with in-hospital mortality were also analyzed.

\section{MATERIALS AND METHODS \\ Setting and Study Population}

National Taiwan University Hospital, a university hospital with a 2200bed capacity, provides both primary and tertiary referral care. It is also an extracorporeal life-support referral center. ${ }^{4,11}$ The first ECMO at this hospital was in August of $1994 .^{3}$ In the following years, ECMO was performed approximately 30 to 60 times per year before 2003 and approximately 100 or more times per year since 2003. A computerized case 


\begin{tabular}{|c|c|}
\hline \multicolumn{2}{|c|}{ Abbreviations and Acronyms } \\
\hline APACH & $\mathrm{I}=$ Acute Physiology and Chronic \\
\hline & Health Evaluation II \\
\hline CI & $=$ confidence interval \\
\hline CPR & $=$ cardiopulmonary resuscitation \\
\hline ECMO & $\begin{aligned}= & \text { extracorporeal membrane } \\
& \text { oxygenation }\end{aligned}$ \\
\hline IABP & $=$ intra-aortic balloon pump \\
\hline OR & $=$ odds ratio \\
\hline VV & $=$ venovenous \\
\hline
\end{tabular}

record form was used to prospectively collect the demographic and clinical data of patients undergoing ECMO, including age, gender, underlying diseases, baseline heart function by New York Heart Association classification, dates of admission, infections before ECMO initiation, and conditions during ECMO use (Acute Physiology and Chronic Health Evaluation II [APACHE II] at ECMO initiation and dates, locations, indications, modes, complications, and outcomes of ECMO use). We also recorded the requirement of an intra-aortic balloon pump (IABP) or CPR during hospitalization and dates of discharge or in-hospital death.

Patients were eligible for the present study if they (1) were aged 16 years or more; (2) underwent ECMO between January 1, 1996, and December 31, 2007; and (3) underwent ECMO for respiratory or circulatory support. Patients were excluded if (1) ECMO was initiated at other hospitals, (2) ECMO use was less than 48 hours because of inadequate ECMO exposure for evaluation of infections during ECMO use, or (3) this was not their firsttime ECMO use. After ECMO initiation, patients were followed until discharge or death during hospitalization. Infections before ECMO initiation were defined as newly emerging or uncontrolled infections within 7 days before ECMO initiation.

Hospital-wide active surveillance of nosocomial infections at National Taiwan University Hospital has been conducted since $1981 .^{12}$ Nosocomial infections were defined as the criteria proposed by the Centers for Disease Control and Prevention definition. ${ }^{13}$ A computerized case record form was used to prospectively collect the data of patients with nosocomial infections, including age; gender; and dates, sites, and causative pathogens of nosocomial infections. The data of infections occurring in the period from the initiation to the removal of ECMO and caused by pathogens different from those of infections within 7 days before ECMO initiation were extracted for analysis. Patients' medical charts were also reviewed to ensure completeness of data collection.

At ECMO initiation, antibiotic prophylaxis with ceftazidime and vancomycin was prescribed to patients without antibiotic use. In patients who had already been taking antibiotics, antimicrobial agents were maintained or modified empirically at the discretion of the attending clinical team. During ECMO use, antibiotics were adjusted according to clinical situations or culture results. The institutional review board of the hospitals approved the study and waived for the need of informed consent.

\section{Statistical Analysis}

All statistical analyses were performed using SPSS software version 16.0 (SPSS Inc, Chicago, Ill). Categoric variables were compared using the chisquare or Fisher's exact test, whereas continuous variables were compared using the Student $t$ test or Mann-Whitney $U$ test and were expressed as mean \pm standard deviation or median with range. A stepwise logistic regression model was built to identify independent variables associated with infections occurring during ECMO use and in-hospital death, respectively. All tests were 2-tailed.

\section{RESULTS}

A total of 569 adults underwent 594 cases of ECMO for respiratory or cardiac support during the study period. Of the 594 cases of ECMO, 260 were excluded for the following reasons: ECMO use for less than 48 hours in 166, ECMO initiated at other hospitals in 62 , and not the first-time use of ECMO in 32. The demographics of the remaining 334 ECMO cases in 334 patients are presented in Table 1. Fifty-five episodes of infection occurred in 45 patients $(13.5 \%, 45 / 334)$ during ECMO use, including 38 episodes $(69.1 \%, 38 / 55)$ of bloodstream infections, $6(10.9 \%, 6 / 55)$ surgical site infections, $4(7.3 \%, 4 / 55)$ respiratory tract infections, $3(5.5 \%, 3 / 55)$ urinary tract infections, $2(3.6 \%$, $2 / 55)$ skin and soft tissue infections, and $2(3.6 \%, 2 / 55)$ catheter infections.

A total of 48 pathogens were isolated from the blood, and gram-negative bacteria $(68.8 \%, 33 / 48)$ were most common, followed by gram-positive bacteria $(16.7 \%, 8 / 48)$ and fungi (14.6\%, 7/48) (Table 2). Stenotrophomonas maltophilia, Candida species, and Pseudomonas aeruginosa were the 3 predominant blood isolates; Staphylococcus aureus was the main gram-positive pathogen. The median interval from ECMO initiation to the onset of bloodstream infections was 8 days (range, 2-32 days). At the onset of bloodstream infections, patients had been hospitalized for a median duration of 20 days (range, 2-89 days).

In the 334 patients, the total duration of ECMO use was 61,424 hours (or 2559 days) and overall rate of bloodstream infection was 14.85 episodes per 1000 ECMO days. Rate of bloodstream infection was 13.83, 23.78, 24.45, and 14.65 episodes per 1000 ECMO days in patients undergoing ECMO for more than 2 to 10 days, more than 10 to 20 days, more than 20 to 30 days, and more than 30 days, respectively (Figure 1).

Surgical site infection developed a median of 23 days (range, 8-35 days) after ECMO initiation, and gramnegative bacteria accounted for $70 \%$ of the causative pathogens (Table 2). Respiratory tract infections occurred at a median of 5 days (range, 5-6 days) of ECMO use, and $80 \%$ were caused by gram-negative bacteria. Urinary tract infection developed on days 10,13, and 13 of ECMO use, respectively. Infections were due to Escherichia coli in 1 episode and yeast-organisms in 2 episodes. Skin and soft tissue infection occurred on days 2 and 22 of ECMO use, respectively, and both patients survived. Catheter infection developed in 2 patients on days 6 and 14 of ECMO use.

Of the 9 isolates of $S$ maltophilia, $11.1 \%(1 / 9)$ were resistant to trimethoprim/sulfamethoxazole, $28.5 \%(2 / 7)$ were resistant to ticarcillin/clavulanate, $33.3 \%$ (3/9) were nonsusceptible to ciprofloxacin, $44.4 \%$ (4/9) were nonsusceptible to ceftazidime, and all were nonsusceptible to cefepime ( $9 /$ 9). Of the 6 isolates of $P$ aeruginosa, none were resistant to gentamicin; $16.7 \%(1 / 6)$ were resistant to ciprofloxacin; $20 \%(1 / 5)$ were resistant to piperacillin/tazobactam; $33.3 \%$ (2/6) were resistant to ceftazidime, cefepime, or aztreonam; 
TABLE 1. Demographic comparison between 45 patients with and 289 patients without infections occurring during extracorporeal membrane oxygenation use

\begin{tabular}{|c|c|c|c|c|}
\hline Characteristics & Total & Infections & No infections & $P$ value \\
\hline Patient no. & 334 & 45 & 289 & \\
\hline Men, \% (n) & $68.0(227)$ & $73.3(33)$ & $67.1(194)$ & .407 \\
\hline Age, y (mean \pm SD) & $51 \pm 17$ & $47 \pm 15$ & $52 \pm 17$ & .099 \\
\hline $16-35$ y, \% (n) & $21.6(72)$ & $28.9(13)$ & $20.4(59)$ & .140 \\
\hline $36-55 y$ & $35.0(117)$ & $35.6(16)$ & $34.9(101)$ & \\
\hline $56-75 y$ & $38.6(129)$ & $35.6(16)$ & $39.1(113)$ & \\
\hline$\geq 76 y$ & $4.8(16)$ & $0(0)$ & $5.5(16)$ & \\
\hline \multicolumn{5}{|l|}{ Underlying diseases, \% (n) } \\
\hline Coronary artery disease & $45.2(151)$ & $40.0(18)$ & $46.0(133)$ & .450 \\
\hline Hypertension & $28.7(96)$ & $28.9(13)$ & $28.7(83)$ & .981 \\
\hline Diabetes mellitus & $26.3(88)$ & $26.7(12)$ & $26.3(76)$ & .958 \\
\hline Valvular heart disease & $13.2(44)$ & $8.9(4)$ & $13.8(40)$ & .361 \\
\hline Cardiomyopathy & $11.1(37)$ & $11.1(5)$ & $11.1(32)$ & .999 \\
\hline Transplantation & $9.3(31)$ & $6.7(3)$ & $9.7(28)$ & .709 \\
\hline Cerebrovascular accident & $6.9(23)$ & $4.4(2)$ & $7.3(21)$ & .705 \\
\hline Malignancy & $6.6(22)$ & $8.9(4)$ & $6.2(18)$ & .729 \\
\hline Congenital heart disease & $4.5(15)$ & $4.4(2)$ & $4.5(13)$ & .999 \\
\hline End-stage renal disease & $4.2(14)$ & $8.9(4)$ & $3.5(10)$ & .197 \\
\hline Autoimmune disease* & $3.0(10)$ & $11.1(5)$ & $1.7(5)$ & .003 \\
\hline Others $\dagger$ & $7.2(24)$ & $13.3(6)$ & $6.2(18)$ & .160 \\
\hline \multicolumn{5}{|l|}{ Indication of ECMO use, $\%$ (n) } \\
\hline Respiratory support & $20.4(68)$ & $37.8(17)$ & $17.6(51)$ & .002 \\
\hline Infection associated & $60.3(41 / 68)$ & $70.6(12 / 17)$ & $56.9(29 / 51)$ & .317 \\
\hline Non-infection associated & $39.7(27 / 68)$ & $29.4(5 / 17)$ & $43.1(22 / 51)$ & \\
\hline Cardiac support & $79.6(266)$ & $62.2(28)$ & $82.4(238)$ & .002 \\
\hline Postcardiotomy $\ddagger$ & $30.1(80 / 266)$ & $17.9(5 / 28)$ & $31.5(75 / 238)$ & .136 \\
\hline Acute myocardial infarction & $29.7(79 / 266)$ & $32.1(9 / 28)$ & $29.4(70 / 238)$ & .765 \\
\hline Cardiomyopathy & $15.4(41 / 266)$ & $28.6(8 / 28)$ & $13.9(33 / 238)$ & .078 \\
\hline Acute myocarditis & $10.5(28 / 266)$ & $3.6(1 / 28)$ & $11.3(27 / 238)$ & .346 \\
\hline $\mathrm{PH}$ and $\mathrm{RH}$ failure & $2.3(6 / 266)$ & $10.7(3 / 28)$ & $1.3(3 / 238)$ & .017 \\
\hline Septic shock & $3.0(8 / 266)$ & $7.1(2 / 28)$ & $2.5(6 / 238)$ & .201 \\
\hline Others $\S$ & $9.0(24 / 266)$ & $0(0 / 28)$ & $10.1(24 / 238)$ & .158 \\
\hline
\end{tabular}

$S D$, Standard deviation; $E C M O$, extracorporeal membrane oxygenation; $P H$, pulmonary hypertension; $R H$, right heart. *Autoimmune disease included systemic lupus erythematosus, Wegener's granulomatosis, dermatomyositis, antiphospholipid syndrome, and scleroderma with vasculitis. †Others included trauma, primary pulmonary hypertension, liver cirrhosis, and chronic obstructive pulmonary disease. $\ddagger$ Postcardiotomy = ECMO use for postcardiotomy cardiogenic shock in patients undergoing cardiac operations, including coronary bypass grafting in $40 \%$ (32/80), heart transplantation in $25 \%(20 / 80)$, heart valvular operations in $18.8 \%$ (15/80), and others in $16.2 \%$ (13/80, including pericardiotomy/ pericardiectomy, correction of congenital heart disease, aortic operation, and pulmonary artery endarterectomy). §Others included acute rejection, congenital heart disease, and unidentified cardiac diseases.

and $40 \%(2 / 5)$ were resistant to carbapenem. Of the 5 isolates of Acinetobacter baumannii, 60\% (3/5) were nonsusceptible to carbapenem; $80 \%(4 / 5)$ were nonsusceptible to ceftazidime, cefepime, or ciprofloxacin; and all (5/5) were resistant to gentamicin.

Of the 21 isolates of enteric bacteria that included 7 Enterobacter species, 6 Klebsiella pneumoniae, 6 E coli, 1 Aeromonas caviae, and 1 Serratia marcescens, 62\% (13/21) were nonsusceptible to cefotaxime, $33.3 \%(7 / 21)$ were resistant to gentamicin, $19 \%(4 / 21)$ were resistant to fluoroquinolone, $14.3 \%(3 / 21)$ were resistant to cefepime, and none $(0 / 21)$ were resistant to carbapenem. Of the 7 isolates of $S$ aureus, $85.7 \%$ (6/7) were resistant to oxacillin and gentamicin, and $42.9 \%$ (3/7) were resistant to trimethoprim/ sulfamethoxazole. All 3 isolates of coagulase negative staphylococci were resistant to oxacillin, and the 2 isolates of Enterococcus faecalis were both susceptible to penicillin.

Compared with patients without infections occurring during ECMO use, those with infections were more likely to have autoimmune disease as the underlying disease $(11.1 \%$ vs $1.7 \%, P=.003)$; to undergo ECMO for respiratory support ( $37.8 \%$ vs $17.6 \%, P=.002)$ or pulmonary hypertension and right-sided heart failure as indication of cardiac support $(10.7 \%$ vs $1.3 \%, P=.017)$; to have infections before ECMO initiation ( $37.8 \%$ vs $17.0 \%, P=.001)$; to receive venovenous (VV) mode $(37.8 \%$ vs $14.0 \%$, $P<.001)$; to have a longer duration of ECMO use (360 \pm 237 vs $157 \pm 165$ hours, $P<.001$ ); and to develop mechanical $(91.1 \%$ vs $56.4 \%, P<.001)$ or pulmonary complications $(17.8 \%$ vs $5.2 \%, P=.005)$ (Tables 1 and 3$)$. 
TABLE 2. Isolates of bloodstream, surgical site, respiratory tract, and urinary tract infections during extracorporeal membrane oxygenation use

\begin{tabular}{|c|c|c|c|c|c|c|c|}
\hline BSI (48 isolates) & $\%($ n) & SSI (10 isolates) & $\%(\mathbf{n})$ & RTI (5 isolates) & $\%(n)$ & UTI (3 isolates) & $\%($ n) \\
\hline Gram-negative bacteria & $68.8(33)$ & & $70.0(7)$ & & $80(4)$ & & $33.3(1)$ \\
\hline Stenotrophomonas maltophilia & $16.7(8)$ & Enterobacter cloacae & $30.0(3)$ & $\begin{array}{l}\text { Burkholderia cepacia } \\
\text { complex }\end{array}$ & $40.0(2)$ & E coli & $33.3(1)$ \\
\hline Pseudomonas aeruginosa & $12.5(6)$ & Klebsiella oxytoca & $10.0(1)$ & $\begin{array}{l}\text { Sphingomonas } \\
\text { paucimobilis }\end{array}$ & $20.0(1)$ & & \\
\hline Klebsiella pneumoniae & $10.4(5)$ & E coli & $10.0(1)$ & Serratia marcescens & $20.0(1)$ & & \\
\hline Acinetobacter baumannii & $10.4(5)$ & Aeromonas caviae & $10.0(1)$ & & & & \\
\hline Enterobacter species & $8.3(4)$ & $S$ maltophilia & $10.0(1)$ & & & & \\
\hline Escherichia coli & $6.3(3)$ & & & & & & \\
\hline Burkholderia cepacia complex & $2.1(1)$ & & & & & & \\
\hline Bacteroides thetaiotaomicron & $2.1(1)$ & & & & & & \\
\hline Gram-positive bacteria & $16.7(8)$ & & $20.0(2)$ & & $20(1)$ & & $0(0)$ \\
\hline Staphylococcus aureus & $8.3(4)$ & $S$ aureus & $20.0(2)$ & $S$ aureus & $20.0(1)$ & & \\
\hline Enterococcus faecalis & $4.2(2)$ & & & & & & \\
\hline Coagulase-negative staphylococci & $4.2(2)$ & & & & & & \\
\hline Fungi & $14.6(7)$ & & $10.0(1)$ & & $0(0)$ & & $66.7(2)$ \\
\hline Candida albicans & $8.3(4)$ & Candida parapsilosis & $10.0(1)$ & & & $\begin{array}{l}\text { Yeast-like } \\
\text { organism }\end{array}$ & $66.7(2)$ \\
\hline Candida tropicalis & $4.2(2)$ & & & & & & \\
\hline Candida glabrata & $2.1(1)$ & & & & & & \\
\hline
\end{tabular}

BSI, Bloodstream infection; SSI, surgical site infection; RTI, respiratory tract infection; UTI, urinary tract infection.

A stepwise logistic regression model was constructed to determine the independent role of duration of ECMO use, infection before ECMO initiation, ECMO use as respiratory support, receipt of VV mode, development of mechanical or pulmonary complications, and underlying disease with autoimmune disease as contributors to occurrence of infections during ECMO use (Table 4). Longer duration of ECMO use (odds ratio [OR], 1.003; 95\% confidence interval [CI], 1.001-1.005; $P=.004]$, mechanical complication
(OR, 4.849; 95\% CI, 1.569-14.991; $P=.006$ ), receipt of VV mode (OR, 4.473; 95\% CI, 1.001-19.977; $P=.050)$, and autoimmune disease (OR, 6.997; 95\% CI, 1.54131.766; $P=.012$ ) were independently associated with a higher risk for infections occurring during ECMO use (Table 4). Infections before ECMO initiation, ECMO use for respiratory support, and development of pulmonary complications were not independently associated with occurrence of infections during ECMO use.



FIGURE 1. Rates of bloodstream infection among patients undergoing ECMO who were stratified by duration of ECMO. ECMO, Extracorporeal membrane oxygenation. 
TABLE 3. Comparison of extracorporeal membrane oxygenation use between patients with and without infections occurring during extracorporeal membrane oxygenation use

\begin{tabular}{|c|c|c|c|c|}
\hline & Total & Infections & No infections & $P$ value \\
\hline Patient no. & 334 & 45 & 289 & \\
\hline IABP during hospitalization, $\%$ (n) & $58.4(195)$ & $46.7(21)$ & $60.2(174)$ & .086 \\
\hline CPR during hospitalization, $\%$ (n) & $49.4(165)$ & $40.0(18)$ & $50.9(147)$ & .175 \\
\hline Infection before ECMO initiation, $\%$ (n) & $19.8(66)$ & $37.8(17)$ & $17.0(49)$ & .001 \\
\hline \multicolumn{5}{|l|}{ Severity of disease at ECMO initiation } \\
\hline APACHE II score, mean \pm SD & $17 \pm 7$ & $16 \pm 7$ & $17 \pm 7$ & .504 \\
\hline$\geq 20, \%$ (n) & $31.4(105)$ & $28.9(13)$ & $31.8(92)$ & .692 \\
\hline $\begin{array}{l}\text { Duration of hospitalization before } \\
\text { ECMO initiation, } d \text {, mean } \pm S D\end{array}$ & $11.3 \pm 26.0$ & $13.2 \pm 17.8$ & $11.0 \pm 27.0$ & .595 \\
\hline ECMO use at admission, $\%(n)$ & $26.9(90)$ & $17.8(8)$ & $28.4(82)$ & .136 \\
\hline$\leq 1$ wk of hospitalization & $68.3(228)$ & $57.8(26)$ & $69.9(202)$ & .134 \\
\hline$\leq 2$ and $>1$ wk of hospitalization & $10.2(34)$ & $8.9(4)$ & $10.4(30)$ & \\
\hline$>2$ wk of hospitalization & $21.6(72)$ & $33.3(15)$ & $19.7(57)$ & \\
\hline \multicolumn{5}{|l|}{ Mode of ECMO, \% (n) } \\
\hline Venoarterial & $80.3(265 / 330)$ & $60.0(27 / 45)$ & $83.5(238 / 285)$ & $<.001$ \\
\hline Venovenous & $17.3(57 / 330)$ & $37.8(17 / 45)$ & $14.0(40 / 285)$ & $<.001$ \\
\hline Duration of ECMO, h, mean $\pm \mathrm{SD}$ & $184 \pm 188$ & $360 \pm 237$ & $157 \pm 163$ & $<.001$ \\
\hline$\leq 184 \mathrm{~h}$ and $>48 \mathrm{~h}, \%$ (n) & $69.2(231)$ & $26.7(12)$ & $75.8(219)$ & $<.001$ \\
\hline$>184 \mathrm{~h}$ & $30.8(103)$ & $73.3(33)$ & $24.2(70)$ & \\
\hline \multicolumn{5}{|l|}{ Complications, \% (n) } \\
\hline Mechanical* & $61.1(204)$ & $91.1(41)$ & $56.4(163)$ & $<.001$ \\
\hline Hemorrhage $\dagger$ & $49.4(165)$ & $62.2(28)$ & $47.4(137)$ & .064 \\
\hline Pulmonary $\ddagger$ & $6.9(23)$ & $17.8(8)$ & $5.2(15)$ & .005 \\
\hline Cardiac tamponade & $9.9(33)$ & $4.4(2)$ & $10.7(31)$ & .296 \\
\hline Vascular§ & $41.6(139)$ & $37.8(17)$ & $42.2(122)$ & .574 \\
\hline \multicolumn{5}{|l|}{ Outcomes of ECMO, \% (n) } \\
\hline ECMO removal and discharge & $27.8(93)$ & $24.4(11)$ & $28.4(82)$ & .075 \\
\hline Bridge to VAD or heart transplantation & $7.5(25)$ & $2.2(1)$ & $8.3(24)$ & \\
\hline Death with ECMO use & 40.7 (136) & $57.8(26)$ & $38.1(110)$ & \\
\hline Death after ECMO removal & $24.0(80)$ & $15.6(7)$ & $25.3(73)$ & \\
\hline Death, \% (n) & $68.3(228)$ & $75.6(34)$ & 67.1 (194) & .259 \\
\hline$\leq 1$ wk of ECMO initiation & $38.6(88 / 228)$ & $23.5(8 / 34)$ & $41.2(80 / 194)$ & .147 \\
\hline$\leq 2$ and $>1$ wk of ECMO initiation & $26.3(60 / 228)$ & $32.4(11 / 34)$ & $25.3(49 / 194)$ & \\
\hline$>2$ wk of ECMO initiation & $35.1(80 / 228)$ & $44.1(15 / 34)$ & $33.5(65 / 194)$ & \\
\hline
\end{tabular}

$I A B P$, Intra-aortic balloon pump; $C P R$, cardiopulmonary resuscitation; $E C M O$, extracorporeal membrane oxygenation; $S D$, standard deviation; $V A D$, ventricular assist device. *Mechanical complications included oxygenator failure; pump malfunction; heater dysfunction; clot of oxygenator, hemofilter, and other sites; connector crack; and so forth. $\dagger$ Hemorrhage complications included gastrointestinal hemorrhage, cannulation site bleeding, surgical site bleeding, and hemolysis. $\ddagger$ Pulmonary complications included pneumothorax and pulmonary hemorrhage. §Vascular complications included digital gangrene, dysfunction of reperfusion catheter, and fasciotomy.

\section{Outcome}

At the end of follow-up, overall mortality was $68.3 \%$ : $75.6 \%$ in patients with infections and $67.1 \%$ in patients without infections occurring during ECMO use $(P=.259)$ (Table 4). The death rate of patients with bloodstream, surgical site, and respiratory tract infections was $88.2 \%$ (30/ $34), 83.3 \%$ (5/6), and $75.0 \%$ (3/4), respectively. Compared with patients who survived, patients who died at the end of follow-up were older and more likely to have coronary artery disease or diabetes mellitus; to undergo ECMO with the indication of cardiac support for acute myocardial infarction; to require CPR during hospitalization; to initiate ECMO in the second week of hospitalization; to have higher APACHE II score; and to have neurologic, metabolic, or vascular complications (Tables E1 and E2). In addition, patients who died were less likely to have cardiomyopathy as the underlying disease, to have a baseline heart function of New York Heart Association class I, to have acute myocarditis as an indication of ECMO support, or to undergo ECMO in the first week of hospitalization than patients who survived (Tables E1 and E2). Infections before or during ECMO use were not associated with in-hospital mortality.

By using stepwise logistic regression analysis with variables in Tables E1 and E2 significant at $P$ less than .05 in the model (ie, age, underlying diseases, baseline heart function, ECMO indications, CPR during hospitalization, APACHE II score, duration of hospitalization before ECMO initiation, and complications of ECMO use), only older age (OR, 1.037; 95\% CI, 1.021-1.054; $P<.001$ ), neurologic complications (OR, 51.153; 95\% CI, 6.773-386.329; 
TABLE 4. Variables associated with infections occurring during extracorporeal membrane oxygenation use by stepwise logistic regression model

\begin{tabular}{|c|c|c|c|}
\hline Variables & Reference & OR $(95 \%$ CI $)$ & $P$ value \\
\hline Duration of ECMO use & Continuous variable & $1.003(1.001-1.005)$ & .004 \\
\hline Infections before ECMO initiation & No infections before ECMO initiation & $1.782(0.721-4.402)$ & .211 \\
\hline Respiratory support & Cardiac support & $0.350(0.072-1.688)$ & .191 \\
\hline Venovenous mode & Venoarterial mode & $4.473(1.001-19.977)$ & .050 \\
\hline Mechanical complication* & No mechanical complication & $4.849(1.569-14.991)$ & .006 \\
\hline Pulmonary complication $\dagger$ & No pulmonary complication & $1.694(0.545-5.264)$ & .362 \\
\hline 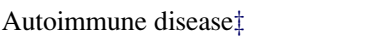 & No autoimmune disease & $6.997(1.541-31.766)$ & .012 \\
\hline
\end{tabular}

$O R$, Odds ratio; $C I$, confidence interval; $E C M O$, extracorporeal membrane oxygenation. * Mechanical complications included oxygenator failure; pump malfunction; heater dysfunction; clot of oxygenator, hemofilter, and other sites; connector crack; and so forth. †Pulmonary complications included pneumothorax and pulmonary hemorrhage. $\ddagger$ †utoimmune disease included systemic lupus erythematosus, Wegener’s granulomatosis, dermatomyositis, antiphospholipid syndrome, and scleroderma with vasculitis.

$P<.001$ ), and vascular complications (OR, $1.922 ; 95 \% \mathrm{CI}$, $1.112-3.320 ; P<.001$ ) were independently associated with a higher risk for death, whereas ECMO initiation within 1 week of hospitalization (OR, $0.291 ; 95 \%$ CI, $0.160-0.530$; $P<.001$ ) was significantly associated with a lower risk for mortality. Underlying diseases, baseline heart function, indications of ECMO use, CPR during hospitalization, and higher APACHE II score were not independently associated with death at the end of follow-up.

\section{DISCUSSION}

In the literature, incidence of infections occurring during ECMO use ranges widely from $3.5 \%$ to $45.1 \%,{ }^{8-10,14,15}$ and bloodstream infection is most common, occurring in $32.6 \%$ to $89.4 \%$ of these infections. ${ }^{10,15,16}$ Gram-positive cocci, particularly coagulase-negative staphylococci, are reported to be the predominant blood isolates. ${ }^{10,16-18}$ Likewise, $13.5 \%$ of our patients developed infections during ECMO use, and $69.1 \%$ of these infections were bloodstream infection. However, $S$ maltophilia and Candida species were the most common blood isolates. Because a single blood isolate of coagulase-negative staphylococci without compatible clinical conditions defined by the Centers for Disease Control was not regarded as a bloodstream infection, this may explain why coagulase-negative staphylococci were not the most common blood isolate in our study. In addition, our practice of antibiotic prophylaxis with vancomycin may contribute to this finding.

$R$ isk factors for infections caused by $S$ maltophilia and Candida species include prolonged hospitalization or intensive care unit stay, ${ }^{19-21}$ exposure to broad-spectrum antibiotics, ${ }^{20-23}$ and use of central venous catheters. ${ }^{21-23}$ Thus, it is not surprising that $S$ maltophilia and Candida species were the predominant blood isolates in this study, and significant proportions of causative pathogens of infections during ECMO use had antibiotic resistance given the fact that our patients with infections during ECMO use had a mean duration of hospitalization of 13.2 days before ECMO initiation. In addition, $37.8 \%$ of them had infections before ECMO initiation, $40.0 \%$ required CPR, and $46.7 \%$ required IABP. These clinical conditions further predisposed patients to prolonged hospital or intensive care unit stay, broad-spectrum antibiotic exposure, and placement of multiple central catheters, which subsequently led to increased risk of acquiring infections caused by resistant organisms.

Given highly invasive procedures (eg, ECMO or IABP) in emergency situations (eg, sudden collapse or worsening respiratory conditions despite full ventilator support), compliance of infection-control precautions might not be perfect. In addition, immunologic responses of patients with sepsis may be inappropriate. The use of steroids for acute respiratory distress syndrome or adrenal insufficiency, body temperature control, and multiple blood transfusions after cardiac operations or for coagulopathy during ECMO use can also interfere with the presentations of infection in patients undergoing ECMO. Thus, we routinely prescribed antimicrobial prophylaxis after ECMO initiation, and it is common to escalate antibiotics immediately and empirically whenever infections are suspected. Although these practices might decrease the occurrence of infections during ECMO use, they also contribute to the subsequent infections caused by drug-resistant bacteria and Candida species as a result of antibiotic selection pressure.

Duration of ECMO use and mechanical complications have been reported as risk factors for occurrence of infections during ECMO use, ${ }^{7,8,10,15,16,18,24}$ and we also had similar observations. Our patients with autoimmune disease and receipt of VV mode also had greater likelihood of infections (Table 4). The association between autoimmune disease and infections has not been previously described. Nevertheless, it is plausible that the immunity of these patients was severely compromised by immunosuppressive agents or their diseases, which predispose them to infections during ECMO use. Before ECMO initiation, 8 of our 10 patients with autoimmune disease received intensified immunosuppression with corticosteroid or cyclophosphamide to control their disease exacerbation.

Compared with VV mode, venoarterial mode has long been considered as a more complex cannulation and associated with more complications, such as vascular trauma, systemic embolization, and ischemia. ${ }^{25}$ It is not clear why VV 
mode was associated with a higher risk for infection in the present study. However, because our patients with VV mode were more likely to require respiratory support, have infections before ECMO use, and undergo ECMO longer than those with venoarterial mode (data not shown), it is possible that the characteristics of patients with VV mode rendered them vulnerable to infections, or that other unknown or unassessed factors influenced the risk of infections.

Infections during ECMO use have also been identified as a risk factor for death. ${ }^{7-9,18}$ However, older age, neurologic, and vascular complications, but not infections during ECMO use, were associated with a higher risk for inhospital death in our study. Elderly patients usually had more comorbidities and were more fragile. Therapeutic strategies tended to be supportive in patients in whom neurologic complications developed during ECMO use (eg, intracranial hemorrhage or brain death), which might explain why the likelihood of in-hospital death in older patients or patients with neurologic complications was greater. Because vascular complications included dysfunction of distal reperfusion catheter $(91.4 \%)$, digital gangrene $(12.2 \%)$, and requirement of fasciotomy (4.3\%) (data not shown), the association between vascular complications and mortality might be due to inadequate perfusion of distal limbs and its associated complications.

It should be noted that several studies have described infections occurring during ECMO use in neonatal and pediatric populations, ${ }^{9,14-18}$ whereas in adults, only 1 study explicitly addressed this issue, and its study population was different from ours. ${ }^{10}$ Our patient population was larger (334 patients with a total of 2559 ECMO days vs 71 patients with a total of 799 ECMO days), was older (mean 51 vs 36.0 years in infected group and 33.3 years in uninfected group), was hospitalized longer before ECMO initiation (mean 11.3 vs 2.3 days in infected group and 1.6 days in uninfected group), and underwent ECMO in recent years (1996-2007 vs 1985-1995), primarily for cardiac support (79.6\% vs $9.9 \%)$. Nevertheless, there were less bloodstream infections in the present study in terms of both overall rate (14.85 vs 18.8 episodes per 1000 ECMO days) and rates stratified by the duration of ECMO use $(13.83,23.78,24.45$, and 14.65 vs 9.5, 27.2, 64.5, and 90.9 episodes per 1000 ECMO days for ECMO duration of $>2-10,>10-20,>20$ 30 , and $>30$ days, respectively). These differences may be attributable to advances of ECMO technology and critical care in the current era, different definitions of infections during ECMO use (ours was from ECMO initiation to removal and theirs ${ }^{10}$ was from 24 hours after initiation to 48 hours after removal), and multidiscipline experience in our ECMO team.

\section{Study Limitations}

Several weaknesses of our study deserve to be acknowledged. First, information regarding antibiotic use and dura- tion in patients undergoing ECMO was not available, so we could not evaluate the effects of antibiotics on the occurrence of infections or resistance of causative pathogens. In addition, variables such as surveillance cultures, nutritional support, need for and amount of blood transfusion, and ventilation times were not assessed, so their impact on the incidence of infection in the patients receiving ECMO is beyond the scope of this report. Second, because the lung parenchyma could be affected by patients' underlying pulmonary or cardiac diseases, it is difficult to distinguish whether superimposed respiratory tract infections occurred. Thus, the rate of respiratory tract infections might be underestimated in our surveillance system. Third, data regarding nosocomial infections in intensive care unit patients without ECMO use who had similar disease severity were not available, and this precludes our ability to further analyze the impact of ECMO use on the occurrence of infections. Last, the present study was limited to a single center.

\section{CONCLUSIONS}

Our study showed that infections during ECMO use occurred in $13.5 \%$ of an adult population. Bloodstream infection was most common and primarily caused by S. maltophilia and Candida species. The majority of the causative pathogens carried antimicrobial resistance. We demonstrated substantial advancement of ECMO care in adults in the current era and identified several risk factors associated with occurrence of infections during ECMO use and in-hospital mortality. These warrant prospective randomized studies to confirm our findings.

The authors thank Dr Victor L. Yu of University of Pittsburgh for the review of this manuscript.

\section{References}

1. Patakas DA, Ulan RA, Brownlee RT, King EG. Extracorporeal oxygenation in the adult respiratory-distress syndrome. N Engl J Med. 1972;287:49.

2. Extracorporeal Life Support Organization. ELSO Registry Information. Available at: http://www.elso.med.umich.edu/Registry.html. Accessed October 17, 2009.

3. Ko WJ, Lin CY, Chen RJ, Wang SS, Lin FY, Chen YS. Extracorporeal membrane oxygenation support for adult postcardiotomy cardiogenic shock. Ann Thorac Surg. 2002;73:538-45.

4. Chen YS, Ko WJ, Chi NH, Wu IH, Huang SC, Chen RJ, et al. Risk factor screening scale to optimize treatment for potential heart transplant candidates under extracorporeal membrane oxygenation. Am J Transplant. 2004;4: 1818-25.

5. Chen YS, Yu HY, Huang SC, Chiu KM, Lin TY, Lai LP, et al. Experience and result of extracorporeal membrane oxygenation in treating fulminant myocarditis with shock: what mechanical support should be considered first? J Heart Lung Transplant. 2005;24:81-7.

6. Chen YS, Lin JW, Yu HY, Ko WJ, Jerng JS, Chang WT, et al. Cardiopulmonary resuscitation with assisted extracorporeal life-support versus conventional cardiopulmonary resuscitation in adults with in-hospital cardiac arrest: an observational study and propensity analysis. Lancet. 2008;372:554-61.

7. Meyer DM, Jessen ME, Eberhart RC. Neonatal extracorporeal membrane oxygenation complicated by sepsis. Extracorporeal Life Support Organization. Ann Thorac Surg. 1995;59:975-80.

8. Douglass BH, Keenan AL, Purohit DM. Bacterial and fungal infection in neonates undergoing venoarterial extracorporeal membrane oxygenation: an analysis of the 
registry data of the extracorporeal life support organization. Artif Organs. 1996; 20:202-8.

9. Montgomery VL, Strotman JM, Ross MP. Impact of multiple organ system dysfunction and nosocomial infections on survival of children treated with extracorporeal membrane oxygenation after heart surgery. Crit Care Med. 2000;28: 526-31.

10. Burket JS, Bartlett RH, Vander Hyde K, Chenoweth CE. Nosocomial infections in adult patients undergoing extracorporeal membrane oxygenation. Clin Infect Dis. 1999;28:828-33.

11. Huang SC, Chen YS, Chi NH, Hsu J, Wang CH, Yu HY, et al. Out-of-center extracorporeal membrane oxygenation for adult cardiogenic shock patients. Artif Organs. 2006;30:24-8.

12. Chen YC, Chang SC, Sun CC, Yang LS, Hsieh WC, Luh KT. Secular trends in the epidemiology of nosocomial fungal infections at a teaching hospital in Taiwan, 1981 to 1993. Infect Control Hosp Epidemiol. 1997;18:369-75.

13. Garner JS, Jarvis WR, Emori TG, Horan TC, Hughes JM. CDC definitions for nosocomial infections, 1988. Am J Infect Control. 1988;16:128-40.

14. Coffin SE, Bell LM, Manning M, Polin R. Nosocomial infections in neonates receiving extracorporeal membrane oxygenation. Infect Control Hosp Epidemiol. 1997; 18:93-6.

15. O'Neill JM, Schutze GE, Heulitt MJ, Simpson PM, Taylor BJ. Nosocomial infections during extracorporeal membrane oxygenation. Intensive Care Med. 2001; 27:1247-53.

16. Brown KL, Ridout DA, Shaw M, Dodkins I, Smith LC, O'Callaghan MA, et al. Healthcare-associated infection in pediatric patients on extracorporeal life sup- port: the role of multidisciplinary surveillance. Pediatr Crit Care Med. 2006;7: 546-50.

17. Elerian LF, Sparks JW, Meyer TA, Zwischenberger JB, Doski J, Goretsky MJ, et al. Usefulness of surveillance cultures in neonatal extracorporeal membrane oxygenation. ASAIO J. 2001;47:220-3.

18. Steiner CK, Stewart DL, Bond SJ, Hornung CA, McKay VJ. Predictors of acquiring a nosocomial bloodstream infection on extracorporeal membrane oxygenation. J Pediatr Surg. 2001;36:487-92.

19. Lai CH, Chi CY, Chen HP, Chen TL, Lai CJ, Fung CP, et al. Clinical characteristics and prognostic factors of patients with Stenotrophomonas maltophilia bacteremia. J Microbiol Immunol Infect. 2004;37:350-8.

20. Apisarnthanarak A, Mayfield JL, Garison T, McLendon PM, DiPersio JF, Fraser VJ, et al. Risk factors for Stenotrophomonas maltophilia bacteremia in oncology patients: a case-control study. Infect Control Hosp Epidemiol. 2003; 24:269-74.

21. Pappas PG. Invasive candidiasis. Infect Dis Clin North Am. 2006;20:485-506.

22. Metan G, Hayran M, Hascelik G, Uzun O. Which patient is a candidate for empirical therapy against Stenotrophomonas maltophilia bacteraemia? An analysis of associated risk factors in a tertiary care hospital. Scand J Infect Dis. 2006;38:527-31.

23. Wenzel RP. Nosocomial candidemia: risk factors and attributable mortality. Clin Infect Dis. 1995;20:1531-4.

24. Schutze GE, Heulitt MJ. Infections during extracorporeal life support. J Pediatr Surg. 1995;30:809-12.

25. Maclaren G, Butt W. Extracorporeal membrane oxygenation and sepsis. Crit Care Resusc. 2007;9:76-80. 
TABLE E1. Demographic comparison between patients who died during hospitalization and patients who survived to be discharged

\begin{tabular}{|c|c|c|c|c|}
\hline Characteristics & Total & Death & Survival & $P$ value \\
\hline Patient no. & 334 & 228 & 106 & \\
\hline Men, \% (n) & $68.0(227)$ & $69.3(158)$ & $65.1(69)$ & .443 \\
\hline Age, y $($ mean \pm SD) & $51 \pm 17$ & $54 \pm 17$ & $45 \pm 16$ & $<.001$ \\
\hline $16-35$ y, \% (n) & $21.6(72)$ & $15.8(36)$ & $34.0(36)$ & $<.001$ \\
\hline $36-55$ y & $35.0(117)$ & $34.2(78)$ & $36.8(39)$ & .645 \\
\hline $56-75$ y & $38.6(129)$ & $43.4(99)$ & $28.3(30)$ & .008 \\
\hline$\geq 76$ y & $4.8(16)$ & $6.6(15)$ & $0.9(1)$ & .025 \\
\hline \multicolumn{5}{|l|}{ Underlying diseases, \% (n) } \\
\hline Coronary artery disease & $45.2(151)$ & $52.2(119)$ & $30.2(32)$ & $<.001$ \\
\hline Hypertension & $28.7(96)$ & $32.0(73)$ & $21.7(23)$ & .052 \\
\hline Diabetes mellitus & $26.3(88)$ & $30.3(69)$ & $17.9(19)$ & .017 \\
\hline Valvular heart disease & $13.2(44)$ & $13.6(31)$ & $12.3(13)$ & .738 \\
\hline Cardiomyopathy & $11.1(37)$ & $8.8(20)$ & $16.0(17)$ & .049 \\
\hline Transplantation & $9.3(31)$ & $8.8(20)$ & $10.4(11)$ & .638 \\
\hline Cerebrovascular accident & $6.9(23)$ & $7.9(18)$ & $4.7(5)$ & .286 \\
\hline Malignancy & $6.6(22)$ & $6.6(15)$ & $6.6(7)$ & .993 \\
\hline Congenital heart disease & $4.5(15)$ & $4.4(10)$ & $4.7(5)$ & .999 \\
\hline End-stage renal disease & $4.2(14)$ & $4.8(11)$ & $2.8(3)$ & .580 \\
\hline Autoimmune disease* & $3.0(10)$ & $4.4(10)$ & $0(0)$ & .065 \\
\hline Others $\dagger$ & $7.2(24)$ & $7.5(17)$ & $6.6(7)$ & .779 \\
\hline \multicolumn{5}{|l|}{ Baseline heart function } \\
\hline NYHA class I & $32.5(82 / 252)$ & $27.5(47 / 171)$ & $43.2(35 / 81)$ & .015 \\
\hline NYHA class II & $29.0(73 / 252)$ & $32.2(55 / 171)$ & $22.2(18 / 81)$ & \\
\hline NYHA class III & $18.7(47 / 252)$ & $22.2(38 / 171)$ & $11.1(9 / 81)$ & \\
\hline NYHA class IV & $19.8(50 / 252)$ & $18.1(31 / 171)$ & $23.5(19 / 81)$ & \\
\hline \multicolumn{5}{|l|}{ Indication of ECMO use, $\%$ (n) } \\
\hline Respiratory support & $20.4(68)$ & $20.6(47)$ & $19.8(21)$ & .865 \\
\hline Infection associated & $60.3(41 / 68)$ & $63.8(30)$ & $52.4(11)$ & .373 \\
\hline Non-infection associated & $39.7(27 / 68)$ & $36.2(17)$ & $47.6(10)$ & \\
\hline Cardiac support & $79.6(266)$ & $79.4(181)$ & $80.2(85)$ & .865 \\
\hline Postcardiotomy $\ddagger$ & $30.1(80 / 266)$ & $28.7(52 / 181)$ & $32.9(28 / 85)$ & .485 \\
\hline Acute myocardial infarction & $29.7(79 / 266)$ & $35.4(64 / 181)$ & $17.6(15 / 85)$ & .003 \\
\hline Cardiomyopathy & $15.4(41 / 266)$ & $13.3(24 / 181)$ & $20.0(17 / 85)$ & .156 \\
\hline Acute myocarditis & $10.5(28 / 266)$ & $5.5(10 / 181)$ & $21.2(18 / 85)$ & $<.001$ \\
\hline $\mathrm{PH}$ and $\mathrm{RH}$ failure & $2.3(6 / 266)$ & $2.8(5 / 181)$ & $1.2(1 / 85)$ & .712 \\
\hline Septic shock & $3.0(8 / 266)$ & $3.9(7 / 181)$ & $1.2(1 / 85)$ & .416 \\
\hline Others $\S$ & $9.0(24 / 266)$ & $10.5(19 / 181)$ & $5.9(5 / 85)$ & .221 \\
\hline
\end{tabular}

$S D$, Standard deviation; $N Y H A$, New York Heart Association; $E C M O$, extracorporeal membrane oxygenation; $P H$, pulmonary hypertension; $R H$, right heart. *Autoimmune disease included systemic lupus erythematosus, Wegener's granulomatosis, dermatomyositis, antiphospholipid syndrome, and scleroderma with vasculitis. $\dagger$ Others included trauma, primary pulmonary hypertension, liver cirrhosis, and chronic obstructive pulmonary disease. †Postcardiotomy = ECMO use for postcardiotomy cardiogenic shock in patients undergoing cardiac operations, including coronary bypass grafting in 40\% (32/80), heart transplantation in $25 \%$ (20/80), heart valvular operations in $18.8 \%$ (15/80), and others in $16.2 \%$ (13/80, including pericardiotomy/pericardiectomy, correction of congenital heart disease, aortic operation, and pulmonary artery endarterectomy); in-hospital mortality of patients with postcardiotomy ECMO use and those without was $65 \%(52 / 80)$ and $69.4 \%(129 / 186)$, respectively $(P=.485)$; of 52 patients with postcardiotomy ECMO who died, causes of in-hospital death were worsening heart function in $46.2 \%(24 / 52)$, sepsis after ECMO removal in $13.5 \%(7 / 52)$, bleeding in $11.5 \%$ (6/52), and miscellaneous causes in $28.8 \%$ (15/52, including respiratory failure in 4 , ventricular tachycardia in 3 , unidentifiable causes in 3 , hepatic failure in 2 , acidosis in 1 , compartment syndrome in 1 , and refractory acidosis in 1). $\S$ Others included acute rejection, congenital heart disease, and unidentified cardiac disease 
TABLE E2. Comparison of extracorporeal membrane oxygenation use between patients who died during hospitalization and those who survived to be discharged

\begin{tabular}{|c|c|c|c|c|}
\hline & Total & Death & Survival & $P$ value \\
\hline Patient No. & 334 & 228 & 106 & \\
\hline IABP during hospitalization, $\%$ (n) & $58.4(195)$ & $60.1(137)$ & $54.7(58)$ & .354 \\
\hline CPR during hospitalization, $\%$ (n) & $49.4(165)$ & $54.4(124)$ & $38.7(41)$ & .008 \\
\hline Infection $<1$ wk before ECMO, $\%$ (n) & $19.8(66)$ & $21.9(50)$ & $15.1(16)$ & .144 \\
\hline \multicolumn{5}{|l|}{ Severity of disease at ECMO initiation } \\
\hline APACHE II score, mean \pm SD & $17 \pm 7$ & $18 \pm 7$ & $15 \pm 7$ & $<.001$ \\
\hline$\geq 20, \%(\mathrm{n})$ & $31.4(105)$ & $37.3(85)$ & $18.9(20)$ & .001 \\
\hline $\begin{array}{l}\text { Duration of hospitalization before } \\
\text { ECMO initiation, } \mathrm{d} \text {, mean } \pm \mathrm{SD}\end{array}$ & $11.3 \pm 26.0$ & $12 \pm 24$ & $10 \pm 30$ & .522 \\
\hline ECMO use at admission, $\%$ (n) & $26.9(90)$ & $25.9(59)$ & $29.2(31)$ & .518 \\
\hline$\leq 1$ wk of hospitalization & $68.3(228)$ & $64.0(146)$ & $77.4(82)$ & .015 \\
\hline$\leq 2$ and $>1$ wk of hospitalization & $10.2(34)$ & $13.2(30)$ & $3.8(4)$ & .008 \\
\hline$>2$ wk of hospitalization & $21.6(72)$ & $22.8(52)$ & $18.9(20)$ & .415 \\
\hline \multicolumn{5}{|l|}{ Mode of ECMO, \% (n) } \\
\hline Venoarterial & $80.3(265 / 330)$ & $81.4(184)$ & $77.9(81)$ & .454 \\
\hline Venovenous & $17.3(57 / 330)$ & $15.5(35)$ & $21.2(22)$ & .206 \\
\hline Duration of ECMO, h, mean $\pm \mathrm{SD}$ & $184 \pm 188$ & $185 \pm 191$ & $182 \pm 182$ & .908 \\
\hline$\leq 184 \mathrm{~h}$ and $>48 \mathrm{~h}, \%(\mathrm{n})$ & $69.2(231)$ & $68.4(156)$ & $70.8(75)$ & .667 \\
\hline$>184 \mathrm{~h}$ & $30.8(103)$ & $31.6(72)$ & $29.2(31)$ & \\
\hline \multicolumn{5}{|l|}{ Complications, \% (n) } \\
\hline Infection & $13.5(45)$ & $14.9(34)$ & $10.4(11)$ & .259 \\
\hline Mechanical* & $61.1(204)$ & $62.3(142)$ & $58.5(62)$ & .508 \\
\hline Hemorrhage $\dagger$ & $49.4(165)$ & $52.2(119)$ & $43.4(46)$ & .134 \\
\hline Neurologic $\ddagger$ & $17.7(59)$ & $25.4(58)$ & $0.9(1)$ & $<.001$ \\
\hline Pulmonaryई & $6.9(23)$ & $6.1(14)$ & $8.5(9)$ & .430 \\
\hline Cardiac tamponade & $9.9(33)$ & $11.8(27)$ & $5.7(6)$ & .078 \\
\hline Metabolic $\|$ & $75.1(251)$ & $81.1(185)$ & $62.3(66)$ & $<.001$ \\
\hline Vascular & $41.6(139)$ & $46.1(105)$ & $32.1(34)$ & .016 \\
\hline
\end{tabular}

$I A B P$, Intra-aortic balloon pump; CPR, cardiopulmonary resuscitation; APACHE II, Acute Physiology and Chronic Health Evaluation II; ECMO, extracorporeal membrane oxygenation; $S D$, standard deviation. *Mechanical complications included oxygenator failure; pump malfunction; heater dysfunction; clot of oxygenator, hemofilter, and other sites; connector crack; and so forth. †Hemorrhage complications included gastrointestinal hemorrhage, cannulation site bleeding, surgical site bleeding, and hemolysis. $\ddagger$ Neurologic complications included brain death, seizure, and cerebrovascular accident. §Pulmonary complications included pneumothorax and pulmonary hemorrhage. $\|$ Metabolic complications included blood glucose level $<40 \mathrm{mg} / \mathrm{dL}$ or $>240 \mathrm{mg} / \mathrm{dL}$, serum $\mathrm{pH}<7.2$ or $>7.6$, or serum bilirubin level $>15 \mathrm{mg} / \mathrm{dL}$. ๆVascular complications included digital gangrene, dysfunction of reperfusion catheter, and fasciotomy 